\title{
Changes in TRPC channel expression during postnatal development of cerebellar neurons
}

Huang W.-C. ${ }^{1}$, Young J. S. ${ }^{2}$, Glitsch M.D. ${ }^{1 *}$

1 Sherrington Building, Department of Physiology, Anatomy and Genetics, Oxford University, Parks Road, Oxford OX1 3PT, UK

${ }^{2}$ Department of Pharmacology, Oxford University, Mansfield Road, Oxford OX1 3QT, UK

*Author for correspondence: Dr. Maike D. Glitsch, Sherrington Building, Department of Physiology, Anatomy and Genetics, Oxford University, Parks Road, Oxford OX1 3PT, UK, maike.glitsch@physiol.ox.ac.uk 


\section{Summary}

In the brain, classical (canonical) Transient Receptor Potential (TRPC) channels are thought to be involved in different aspects of neuronal development. We investigated the developmental expression profile of TRPC channels in rat cerebellum during the first 6 weeks after birth. TRPC3 expression is significantly up-regulated whereas TRPC4 and TRPC6 expression are significantly down-regulated over this period of time. TRPC3 expression is mainly found on Purkinje cells and their dendrites, suggesting that the increase in TRPC3 expression reflects development of the dendritic tree of Purkinje cells. TRPC4 expression was restricted to granule and their precursor cells. TRPC6 expression is found on Purkinje cell bodies, on mature granule cells in the internal granule cell layer (but not its precursors) and interneurons in the molecular layer. The decrease in TRPC4 expression suggests that it is required for proper granule cell development whereas the decrease in TRPC6 expression is presumably correlated with interneuron development. Moreover, we demonstrate the presence of functional TRPC channels on Purkinje cell dendrites that are activated following stimulation of metabotropic glutamate receptors. Our results reveal cell-specific expression patterns for different TRPC proteins and suggest that developmental changes in TRPC protein expression may be required for proper postnatal cerebellar development. 


\section{Introduction}

The Transient Receptor Potential (TRP) superfamily of ion channels is widely expressed in eukaryotes. Of the six mammalian TRP subfamilies (TRPA, TRPC, TRPM, TRPML, TRPP, TRPV), the TRPCs (classical or canonical TRP) bear most sequence ho mologies with the original TRP channel cloned from Drosophila photoreceptors [1]. The TRPC subfamily, comprising TRPC1-7, can be divided into four groups based on sequence homologies and functional properties: TRPC1, TRPC2, TRPC3/6/7 and TRPC4/5, although TRPC1 is sometimes placed with TRPC4/5, thus yielding three groups [1]. In humans, TRPC2 is a pseudogene, whereas in rodents it is mainly expressed in the vomeronasal organ and is involved in pheromone sensation [2].

It is widely accepted that all TRPC subunits are somehow activated following phospholipase C (PLC) activation and form cation-selective ion channels. The seven TRPC subunits can form homo- and heteromeric channels and thus generate a whole plethora of channels with distinct properties such as different $\mathrm{Ca}^{2+}$ permeabilities $[1,3]$. Most TRPC subunits are prominently expressed in the CNS. TRPC5 has been implicated in regulating neurite outgrowth of rat hippocampal neurons $[4,5]$, whereas TRPC3 and TRPC6 seem to play a role in brain-derived neurotrophic factor-dependent chemoattractive turning in cerebellar granule cell cultures [6]. TRPC1 is involved in basic fibroblast growth factor (bFGF)-dependent embryonic rat neural stem cell proliferation [7]. All these studies suggest a role for TRPC channels during brain development. However, these studies were primarily based on cultured neurons and hence the relevance to native tissue is unclear.

Of all the regions of the brain, the development of the cerebellum is the best understood. Cerebellar development is unusual in that neurogenesis takes place in two different proliferation zones at different developmental stages. Prenatal neurogenesis in the ventricular zone gives rise to deep cerebellar neurons, Purkinje cells, inhibitory interneurons and glia cells whereas neurogenesis in the external granule cell layer (a prenatally-formed secondary proliferation zone) postnatally generates granule cells [8]. 
At birth, the cerebellar cortex consists of four layers: the external granule cell layer, the molecular layer, the Purkinje cell layer and the internal granule cell layer. Following birth, granule precursor cells in the external granule cell layer start to proliferate, postmitotic granule cells migrate radially inwards and form the internal granule cell layer. This process is complete in rats three weeks after birth and the external granule cell layer disappears [8]. The profile of TRPC channel expression during development is unclear. Here, we have examined the expression patterns of the different TRPC subunits in rat cerebellum for the first six postnatal weeks (i.e. from newborn to young adult) to establish potential links between cellular development and TRPC expression in native tissue. We find striking changes in expression as the cerebellum develops, in a cell-type specific manner. 


\section{Material and Methods}

\section{Tissue and Slices preparation}

Sprague-Dawley rats were killed by cervical dislocation. Cerebella were from rats aged from postnatal days 1 to 42 (P1, P4, P8, P12, P16, P21, P28, and P42). Following removal, cerebella were washed immediately with ice-cold DEPC-PBS. For immunohistochemical staining, cerebella were embedded in Tissue-Tek OCT compound (Sakura Finetek, Torrence, CA), sectioned by cryostat (20 $\mu \mathrm{m}$ serial sagittal sections), and mounted on Fisherbrand Superfrost Plus slides (Fisher Scientific, Pittsburgh, PA).

For electrophysiological and calcium imaging experiments, sagittal cerebellar slices were obtained from Sprague Dawley rats aged 13 days, as previously described [9]. Briefly, rats were decapitated following cervical dislocation and the cerebellar vermis was dissected out. The vermis was cooled in ice-cold bicarbonate-buffered saline (BBS, composition see below) for 2-4 minutes and then glued to the stage of a vibratome. Slices of $200 \mu \mathrm{m}$ thickness were cut and incubated in oxygenated BBS at room temperature for at least 40 minutes before their use in experiments.

\section{Total RNA Isolation}

Total RNA was isolated from whole cerebella and using an RNeasy Mini-Kit (Qiagen,Valencia, CA) and treated with DNase I (Sigma). The RNA concentration was determined by measuring absorbance at $260 \mathrm{~nm}$.

\section{PCR Primers}

PCR primers for rTRPC1-7 were designed based on published sequences in GeneBank ${ }^{\mathrm{TM}}$ (Supplementary Table I). All designed primers were screened using BLAST (Basic Local Alignment Search Tool).

\section{RT-PCR}

First-strand cDNA was prepared from $1 \mu \mathrm{g}$ of total RNA using the Superscript II Kit (Invitrogen, Carlsbad, CA) in the presence of 1 pg of Oligo(dT). Briefly, the mRNA samples were denatured at $65^{\circ} \mathrm{C}$ for $5 \mathrm{~min}$. Reverse transcription was performed at $50^{\circ} \mathrm{C}$ for $55 \mathrm{~min}$ and was stopped by heating the sample at $75^{\circ} \mathrm{C}$ for $10 \mathrm{~min}$. Following cDNA synthesis, PCR was then performed with primers specific for the different TRPCs (see 
Supplementary Table I). 35 cycles of PCR were performed, with each cycle consisting of denaturation at $95^{\circ} \mathrm{C}$ for $30 \mathrm{~s}$, annealing at $50^{\circ} \mathrm{C}$ for $30 \mathrm{~s}$, and extension at $72^{\circ} \mathrm{C}$ for $30 \mathrm{~s}$. Control PCRs were carried out with primers for the housekeeping gene, glyceraldehyde3-phosphate dehydrogenase (GAPDH), as an internal control alongside the experimental samples, as well as a negative control which didn't contain any cDNA. The PCR products were electrophoresed through a $2 \%$ agarose gel and visualized by ethidium bromide staining. The identity of the amplified PCR products was verified by an ABI PRISM DNA sequencing system (Perkin Elmer).

For the semi-quantification, RT-PCR products of the genes of interest, TRPC1,-3,-4,-5,-6 and -7, from P1, P4, P8, P12, P16, P21, P28 and P42 SD rats were grouped and analyzed simultaneously. Three independent PCRs were carried out for each pair of TRPC primers and also for GAPDH primers (internal control), and the PCR products were quantified by Gene Tools (SYNGENE, Cambridge, UK) and expressed as the ratio of TRPC product to GAPDH. The ratio value obtained for PCR primers with cerebellar tissue from P1 was then taken to be 1 (i.e. 100\%) and all other ratios (obtained with the same TRPC primers but at different postnatal days) were normalized to this P1 value.

\section{Protein Lysates Preparation}

After several washes with ice-cold PBS, cerebella were minced and suspended in lysis buffer containing $150 \mathrm{mM} \mathrm{NaCl}, 10 \mathrm{mM}$ Tris- $\mathrm{Cl}(\mathrm{pH}$ 7.5) and $1 \%$ Triton X-100, supplemented with protease inhibitor cocktail (Sigma, St. Louis, MO). The brain suspension was homogenized, and centrifuged at $8000 \mathrm{rpm}$ for $10 \mathrm{~min}$ to remove the tissue debris. The protein lysate was frozen and stored at $-20^{\circ} \mathrm{C}$ until further use.

\section{Western Blotting}

Samples of equal amounts of protein $(\sim 60 \mu \mathrm{g})$ were analyzed by SDS-PAGE on $10 \%$ polyacrylamide gels, then transferred onto a nitrocellulose membrane, which was then incubated in TBST (150 mM NaCl, $20 \mathrm{mM}$ Tris, and 0.02\% Tween, $\mathrm{pH}$ 7.4) containing $1 \%$ nonfat milk for $1 \mathrm{~h}$ at room temperature. Membranes were washed with TBST once and then incubated with primary antibody for $1 \mathrm{~h}$ at room temperature. The following antibodies were used: rabbit anti-TRPC1, anti-TRPC3, anti-TRPC5, anti-RPC6, and TRPC7 (1:1000; generous gifts from Dr. W. Schilling, Physiology and Biophysics, Case Western Reserve University, OH), rabbit anti-TRPC4 (1:500; Chemicon). Then the 
membranes were washed with TBST three times and incubated with 1:2000 dilution of peroxidase-linked anti-mouse IgG (Amersham Biosciences Europe GmbH, Freiburg, Germany) for $1 \mathrm{~h}$ at room temperature. After washing with PBS, the bands were detected by an ECL-plus Western blotting detection system (Amersham Biosciences). For the quantification, films were scanned on a flatbed scanner and the relative band intensities were digitalized and analyzed by using UN-SCAN-IT software (Silk Scientific, UT, USA). Western blots were repeated at least three times using different cell lysates.

\section{Immunohistochemical staining}

Cerebellar slices were fixed in $4 \%$ paraformaldehyde for $20 \mathrm{~min}$ at room temperature, and then incubated overnight at $4{ }^{\circ} \mathrm{C}$ with primary antibodies at the appropriate dilution in PBS containing $1 \%$ bovine serum albumin (BSA). The following antibodies were used: rabbit anti-TRPC1, anti-TRPC3, anti-TRPC5, anti-TRPC6, and TRPC7 (1:1000; generous gifts from Dr. W. Schilling, Physiology and Biophysics, Case Western Reserve University, OH), rabbit anti-TRPC4 (1:500; Chemicon). Sections were washed three times with PBS and incubated with secondary antibodies: Alexa Fluor 488-, Alexa Fluor 594-coupled anti-rabbit or anti- mouse IgG antibodies (Molecular Probes, Eugene, OR) in

a dilution of 1:1000 for $1 \mathrm{~h}$ at room temperature. After washing with PBS for $15 \mathrm{~min}$, the slices were mounted with Vectashield (Vector Laboratories). High resolution images were acquired using a Leica SP2 upright confocal microscope (1024 x 1024 pixels; Leica Microsystems, Milton Keynes, UK; Fig. 4 and 5) in the laboratory of Dr. Tom Cunnane (Department of Pharmacology, University of Oxford) and using a Leica DM IRBE (512 x 512 pixels; Leica Microsystems, Milton Keynes, UK; Fig. 3 and 6) Dr. Peter Kohl (Department of Physiology, Anatomy and Genetics, Oxford University).

\section{Electrophysiology}

Tight-seal whole cell recordings were obtained from Purkinje cells, as described in [10]. A $63 x$ water immersion objective was used for visualization of the slices during experiments. Experiments were performed using an EPC9/2 patch-clamp amplifier (Heka Electronics, Lambrecht, Germany). Patch pipettes had resistances of 2-3 MOhm with the standard internal and extracellular solutions used (composition see below). Series 
resistance did not exceed $10 \mathrm{MOhm}$. Capacitative currents were cancelled and series resistance was compensated by between 50-60\%, as described in [10].

Experiments were performed at room temperature and slices were continuously perfused with oxygenated BBS at a rate of $2 \mathrm{ml} / \mathrm{min}$. Drugs were applied to the slice by dissolving prepared stock solutions into BBS to the desired concentration. Only one cell per slice was used for each experiment to avoid contamination of the results by potential long-term side effects of the drugs used.

\section{Fluorescent $\mathrm{Ca}^{2+}$ imaging experiments}

Experiments were performed simultaneously with electrophysiological experiments. An intracellular solution containing the fluorescent $\mathrm{Ca}^{2+}$ dye Fura- 2 was used for monitoring changes in intracellular $\mathrm{Ca}^{2+}$ concentration (see below). The intracellular $\mathrm{Ca}^{2+}$ concentration was measured with a fluorometric system from T.I.L.L. Photonics (Martinsried, Germany). The system utilizes the Polychrome II monochromator and employs an Imago CCD camera for light detection. Recordings were started 5-6 min after establishing WCR. The CCD camera (640 x 480 pixels) was used for imaging the soma and proximal dendritic arborization. The standard imaging protocol consisted of a sequence of 180 images at both 356 and $380 \mathrm{~nm}$ (for each wavelength $30 \mathrm{~ms}$ exposure; 1 $\mathrm{s}$ interval between 2 pictures of a given wavelength). Acquisition started when electrophysiological recordings started. Fluorescence changes were analysed on-line as ratio of fluorescence measured at $356 \mathrm{~nm}$ over fluorescence measured at $380 \mathrm{~nm}$ $\left(? \mathrm{~F}_{356} / \mathrm{F}_{380}\right)$ in small regions of interest (ROI). Usually one somatic ROI and three ROIs distributed throughout the proximal dendritic tree were measured. One ROI was placed in the periphery of the visual field as a background fluorescence control. For statistical analysis, the three dendritic fluorescent ratio values were averaged to give one dendritic value for a given cell. Basal ? $\mathrm{F}_{356} / \mathrm{F}_{380}$ prior to drug application was compared with peak ? $\mathrm{F}_{356} / \mathrm{F}_{380}$ following drug application for each ROI.

\section{Solutions and Drugs for Electrophysiology and $\mathrm{Ca}^{2+}$ imaging experiments}


The standard external solution BBS contained (in $\mathrm{mM}$ ): $125 \mathrm{NaCl}, 2.5 \mathrm{KCl}, 26 \mathrm{NaHCO}_{3}$, $1.25 \mathrm{NaH}_{2} \mathrm{PO}_{4}, 2 \mathrm{CaCl}_{2}, 1 \mathrm{MgCl}_{2}$ and 10 glucose $(\mathrm{pH} 7.4$ when oxygenated with carbogen).

The intracellular solution contained (in $\mathrm{mM}$ ): $150 \mathrm{CsCl}, 10 \mathrm{HEPES}, 0.4$ Fura-2 (pentapotassium salt), $4 \mathrm{Na}-\mathrm{ATP}, 0.4 \mathrm{Na}-\mathrm{GTP}, 4.6 \mathrm{MgCh}$ ( $\mathrm{pH}$ adjusted to 7.3 with $\mathrm{CsOH})$. Prior to filling the pipette with this solution, pipette tips were briefly dipped into an internal solution identical to that described above but without any Fura-2.

Tetrodotoxin (TTX), 6-Cyano-7-nitroquinoxaline-2, 3-dione (CNQX; disodium salt) and (S)-3, 5-Dihydroxyphenylglycine (DHPG) were obtained from Tocris Cookson (Bristol, UK). Ryanodine and thapsigargin were obtained from Sigma Aldrich (Poole, UK). Ryanodine and thapsigargin, were dissolved in DMSO, CNQX, DHPG and TTX were dissolved in water. Drug stocks were aliquoted and frozen at $-20^{\circ} \mathrm{C}$. All experiments were carried out in the presence of $0.3 \mu \mathrm{M}$ TTX and $10 \mu \mathrm{M}$ CNQX; the DHPG concentration used was $100 \mu \mathrm{M}$.

\section{Statistical Analysis}

Unpaired and paired Student t-tests were used for statistical analysis and differences were considered significant for $P<0.05$. 


\section{Results}

\section{TRPC3 protein expression is increased whereas TRPC4 and TRPC6 protein expression is decreased during postnatal development}

In a first series of experiments, mRNA was isolated from whole cerebella of animals ranging between P1-P42, i.e. newborns till early adulthood. The mRNA was used in RTPCR experiments with primers for all seven rat TRPC channel subunits. Fig. 1A shows RT-PCR results for TRPC1, -3, -4, -5, -6 and -7 for the postnatal ages P1, P4, P8, P12, P16, P21, P28 and P42. Primers for TRPC2 did not give rise to any detectable band, reflecting the fact that TRPC2 is mainly found in the vomeronasal organ [2]. GAPDH was used as an internal control (see Methods).

It can be seen that mRNA for all TRPC subunits (except TRPC2) is expressed right after birth (P1) until early adulthood (P42). The most striking pattern of expression is found for TRPC4 - it steadily declines and is dramatically reduced by P42.

Fig. 1B shows that the relative TRPC4 expression was significantly reduced by $66.2 \% \pm$ $6.1 \%(P=0.0012 ; n=3$; unpaired t-test $)$ when compared with the corresponding value for P1 whereas the changes in expression for the other TRPC subunits were not statistically significant. The decrease in TRPC4 mRNA expression was already significant by P16 when compared to P1 (reduced by $46 \% \pm 9.3 \% ; P=0.0126 ; n=3$; unpaired t-test).

These results suggest that TRPC4 mRNA is continually down-regulated during cerebellar development. However, mRNA levels are not necessarily reliable representatives of actual protein levels and we therefore decided to investigate TRPC channel expression in cerebellar tissue at P1 (newborn), P16 (adolescent) and P42 (young adult) at a protein level using antibodies specific for the different channel proteins in Western blots.

Fig.2A shows that all TRPC subunits that had been found by RT-PCR were confirmed to be present at the protein level at P1, P16 and P42. Antibodies against GAPDH were used as an internal control for Western blots. Fig. 2B shows that TRPC3 protein levels were significantly up-regulated with development (increase between P1 and P42 by $145.4 \% \pm$ 
$6.4 \% ; P<0.0001 ; n=4$; unpaired t-test), while TRPC4 channel protein was significantly down-regulated by $67.1 \% \pm 10.6 \%(P=0.0025 ; n=4$; unpaired t-test $)$. TRPC6 was also significantly down-regulated (by $49.3 \% \pm 5.7 \% ; P=0.0045 ; n=3$; unpaired t-test). For the other TRPC subunits there was no significant change in expression between P1 and P42.

Taken together, the results suggest that TRPC3 subunit levels are up-regulated whereas TRPC4 and TRPC6 subunit levels are down-regulated during postnatal cerebellar development.

\section{TRPC3 subunit expression is mainly found on Purkinje cells}

It is of interest to know where in the cerebellum the different TRPC subunits are expressed and we therefore carried out immunohistochemical experiments in rat cerebellar slices at P1, P16 and P42.

Fig. 3A shows that anti-TRPC3 antibodies gave diffuse staining at P1 but which was most intense in the Purkinje cell layer (PCL). At P16, there was very clear staining of Purkinje cell bodies in the PCL and their dendrites (staining of molecular layer (ML) but not external granule cell layer(EGL)) and some staining of the internal granule cell layer (IGL). At P42, the expression pattern of TRPC3 was very similar to that at P16 extensive staining of Purkinje cell bodies in the PCL and dendrites in the ML but only minor staining of the IGL.

\section{TRPC4 subunit expression patterns mirrors granule cell development}

Fig. 4 shows the expression pattern for TRPC4 which had a very striking expression pattern in that it reflected granule cell development. At P1, TRPC4 is only found in the external granule cell layer (EGL) where granule precursor cells proliferate.

At P16, TRPC4 is found in the EGL, in individual cells in the ML and in the IGL. At this stage, granule precursor cells in the EGL are still present. Post-mitotic granule cells migrate through the ML, which at this stage also contains fully differentiated interneurons (stellate and basket cells). Differentiated granule cells form the IGL (there 
are also some other cell types in the IGL, most notably Golgi cells, but these cells are far outnumbered by granule cells and have larger cell bodies than granule cells).

At P42, granule cell development is complete and there are only fully differentiated granule cells in the IGL, and strikingly TRPC4 staining is restricted to the IGL.

We wanted to provide more direct evidence that the TRPC4 positive cells of the ML are granule cells and not interneurons which can also be found in the ML at P16 and P42. We

therefore used the neuron-specific nuclear protein Neuronal Nuclei (NeuN) which only recognises post-mitotic granule cells within the developing and adult cerebellar cortex $[11,12]$.

Fig. 5 compares NeuN staining patterns at P28 (a developmental stage at which granule cells are only found in the IGL; right panel) with that of TRPC4 at the same age (left panel). It can be clearly seen that both antibodies stain the same cell population. Importantly, there is no appreciable staining of cells in the ML with either antibody, demonstrating that neither of them recognises interneurons which are present in the ML. It is very unlikely that interne urons of the ML should only express TRPC4 at around P16 but not at P28 or P42, given that their development is completed before P16. It therefore seems very likely that the TRPC4 positive cells in the ML at P16 are indeed migrating granule cells.

We also carried out double-staining experiments in which we used antibodies against TRPC4 and NeuN to see if they co-localise. Unfortunately, however, we could not get any clear staining with anti-TRPC4 antibodies in the presence of the anti-NeuN antibody (data not shown).

\section{TRPC6 expression is found on interneurons, Purkinje cells and postmitotic granule cells}

Fig. 6 shows expression patterns for TRPC6. As for TRPC3, at P1 there was very diffuse staining with the anti-TRPC6 antibody. At P16, TRPC6 staining was clearly found on individual cells in the ML, on Purkinje cell bodies in the PCL and on granule cells in the IGL. At P42, TRPC6 staining was identical to that at P16. Since at this stage granule cells can only be found in the IGL, the TRPC6-positive cells in the ML are interneurons 
(basket and stellate cells) and it seems reasonable to argue that most, if not all TRPC6positive cells in the ML at P16 also reflect interneurons and not migrating granule cells.

\section{Purkinje cells show increases in intracellular $\mathrm{Ca}^{2+}$ concentration following stimulation of metabotropic glutamate receptors that are independent of $\mathrm{Ca}^{2+}$ stores and voltage-gated calcium channels and likely reflect $\mathrm{Ca}^{2+}$ influx through TRPC channels}

Purkinje cells are known to express group I metabotropic glutamate receptors (mGluRs) which couple to $\mathrm{Ca}^{2+}$ release from intracellular $\mathrm{Ca}^{2+}$ stores via activation of PLC and subsequent inositol trisphosphate production. As mentioned before, activation of PLC can also lead to activation of TRPC channels. We therefore carried out simultaneous patchclamp and $\mathrm{Ca}^{2+}$ imaging experiments to see if activation of group I mGluRs resulted in activation of TRPC channels. Slices were preincubated for 50-60 min in BBS containing $2 \mu \mathrm{M}$ thapsigargin and $10 \mu \mathrm{M}$ ryanodine. Under these conditions, intracellular $\mathrm{Ca}^{2+}$ stores are depleted [13] and activation of group I mGluRs should no longer trigger $\mathrm{Ca}^{2+}$ release from intracellular $\mathrm{Ca}^{2+}$ stores. Hence, stimulation of group I mGluRs should neither trigger store-operated nor $\mathrm{Ca}^{2+}$-activated conductances. As can be seen in Fig. 7 for one representative Purkinje cell, application of $100 \mu \mathrm{M}$ DHPG, a selective group I mGluR agonist, resulted in the development of a clear inward current in the Purkinje cell (bottom panel) and a simultaneous increase in intracellular $\mathrm{Ca}^{2+}$ concentration (top panel) both in the soma (solid line) and dendrites (dotted line) of the Purkinje cell. On average, the somatic $\mathrm{Ca}^{2+}$ signal was increased from a basal $? \mathrm{~F}_{356} / \mathrm{F}_{380}$ of $568 \pm 52$ to $625 \pm 53$ units (significant increase: $P=0.043 ; n=3$; paired t-test), whereas the dendritic $\mathrm{Ca}^{2+}$ signal was increased from $? \mathrm{~F}_{356} / \mathrm{F}_{380} 638 \pm 10$ to $729 \pm 9$ units (significant increase: $P=0.007$; $n=3$; paired t-test).

Since the inward current and increase in intracellular $\mathrm{Ca}^{2+}$ concentration were observed following activation of PLC, it is likely that they reflect activation of TRPC channels. 


\section{Discussion}

The main finding of this study is that different cell types in the cerebellar cortex express different TRPC subunits (or combinations thereof) and that their expression levels can significantly change during postnatal development. To our knowledge, this is the first demonstration of changes in TRPC expression during brain development in native tissue.

One major concern with immunohistochemical studies is the specificity of the antibody used. We used Professor Schilling's antibodies against TRPC1, TRPC3, TRPC5, TRPC6 and TRPC7, which are generally accepted to be very reliable. We used a commercial anti-TRPC4 antibody since Professor Schilling's anti-TRPC4 antibody did not give rise to any detectable bands in the Western blots or any staining in rat cerebellar slices, presumably reflecting the fact that the TRPC4ß splice variant [14] of the TRPC4 subunit is expressed in rat cerebellum (Professor Schilling's antibody recognises only the TRPC4a subunit; also see below). Importantly, we find TRPC4 down-regulation both at the mRNA and protein level, suggesting that the anti-TRPC4 antibody does indeed recognise TRPC4.

We find that TRPC3 subunits are mainly expressed on cerebellar Purkinje cell bodies and dendrites whereas TRPC4 subunit expression is restricted to granule (precursor) cells. TRPC6 expression is found on interneurons, Purkinje cells and post-mitotic granule cells in the IGL (possibly also on migrating granule cells but not granule precursor cells).

Interestingly, the level of expression of these TRPC subunits changes significantly during development.

TRPC3 subunit expression is significantly up-regulated and Fig. 2 shows that the main increase in TRPC3 expression is between P1 and P16 (increase by $97.7 \% \pm 5.4 \% ; P=$ 0.0002; $n=4$; for comparison: only $24.2 \%$ further increase between P16 and P42). The maturation of Purkinje cell dendritic trees occurs mainly between P10 and P15; they 
reach their adult width by $\mathrm{P} 15$ and from P15 onwards only vertical growth continues which is complete by about P30 [8]. Correspondingly, the main increase in TRPC3 expression is found between P1 and P16, suggesting that there is a tight correlation between increase in TRPC3 expression and maturation of Purkinje cell dendritic trees. It is important to note that the final number of Purkinje cells is present at birth; hence the increase in TRPC3 expression does not reflect an increase in Purkinje cell number.

For TRPC4, the expression pattern was particularly striking. Already at P1, it was restricted to the EGL containing granule precursor cells and did not show any of the diffuse staining observed for TRPC3 and TRPC6. At P16, it mirrored granule cell development with staining of the EGL (granule precursor cells), staining of individual cell in the ML (migrating granule cells) and staining of the IGL (mature granule cells). At P42 (and also P28, Fig. 5), when granule cells can only be found in the IGL, TRPC4 expression was restricted to that layer. These results suggest that TRPC4 down-regulation goes in parallel with granule cell development.

It is interesting to note that the main decrease in TRPC4 expression occurs between P1 and P16, when most proliferating granule precursor cells exit the cell cycle and migrate to their final destination, the IGL. In rats, granule cell development is complete 3 weeks after birth, when the EGL disappears, and the fact that the main decrease in TRPC4 expression occurs between P1 and P16 suggests that TRPC4 down-regulation goes along with the initial stages of granule cell differentiation and/or migration. It should be noted that granule cells are the most abundant neuron in the brain - around $50 \%$ of all neurons in the brain are cerebellar granule cells. Hence, the $67 \%$ decrease in TRPC4 expression is an underestimate of the actual decrease in TRPC4 expression at an individual granule cell level, since TRPC4 expression decreases as granule cell number increases.

For TRPC6, it is much harder to correlate the decrease in TPRC6 expression with the development of the cerebellar cortex since it is found in a number of different cell types: granule cells, Purkinje cells and interneurons. However, the fact that there is no significant difference between TRPC6 expression at P16 and P42 seems to suggest that TRPC6 down-regulation goes in parallel with interneuron development (which is 
complete 2 weeks after birth in rats). It is possible that an increase in TRPC6 expression in granule cells and/or Purkinje cells is masked by a more marked decrease in TRPC6 expression following completion of interneuron development.

The presence of TRPC1, 3, 6, and 7 in rat cerebellum had previously been reported [6, 15-17]. This is the first study to show that both TRPC4 and TRPC5 are also expressed in rat cerebellum. Two previous studies did not find any TRPC4 or TRPC5 expression in rat cerebellum $[6,16]$.

Li et al. (2005) reported an essential role for both TRPC3 and TRPC6 in the guidance of nerve growth cones of cultured cerebellar granule cells by BDNF but did not find any substantial TRPC4 expression in either cultured rat cerebellar granule cells or in whole rat cerebella at the mRNA level. This latter result conflicts with our findings.

It is important to note that $\mathrm{Li}$ et al. (2005) used granule cell cultures and under their conditions, the granule cell culture may have been in a differentiating rather than proliferating state and hence TRPC4 expression would be greatly reduced. This could also explain the presence of TRPC3 and TRPC6 in granule cells which we only found in post-mitotic granule cells but not their precursors.

The fact that Li et al. (2005) did not find TRPC4 expression in whole rat cerebella may reflect the fact that they did not compare cerebellar tissue from rats of different ages and the age of the rats they used is unclear. Hence, it could be that TRPC4 mRNA levels were too low to generate sufficient amounts of PCR product or that any bands on the gel following RT-PCR were too faint to be detected. We used the TRPC4 primers described in Li et al. (2005) in an RT-PCR experiment with cerebellar tissue from P12 and P28 and in both cases we found an appropriate band for the TRPC4 primers which subsequent sequencing verified to be TRPC4.

Li et al. (2005) also did not find appreciable TRPC5 or TRPC7 mRNA expression in either cerebellar granule cell cultures or whole cerebella. TRPC7 protein expression had previously been shown to be present in rat cerebellum by Goel et al. (2002), a finding which is confirmed in the present study at both mRNA and protein level. Mizuno et al. (1999) did also not find any TRPC4 or TRPC5 expression using cerebellar RNA isolated from rats at $\mathrm{P} 21$. 
The reasons for these discrepancies are unclear but likely reflect differences in PCR protocols and/or primers used. Interestingly, the primers for TRPC4 used by Mizuno et al. (1999) only recognise the TRPC4a splice variant [14], which is presumably not expressed in cerebellum (see above), and it should be noted that TRPC5 has been shown to be expressed in human cerebellum [18].

TRPC channels form non-selective cation channels that can have a high permeability to $\mathrm{Ca}^{2+}$ [19], suggesting that $\mathrm{Ca}^{2+}$ permeability through TRPC channels is likely to increase cytosolic $\mathrm{Ca}^{2+}$ levels significantly. Changes in intracellular $\mathrm{Ca}^{2+}$ concentration are known to be involved in such key processes as neuronal cell proliferation, differentiation, migration and cell death and hence it is tempting to speculate that the expression of the different TRPC subunits is required for proper cellular development. In the case of TRPC4 and TRPC6, their down-regulation may be required for initiation of differentiation and/or migration of granule cells (TRPC4) or interneurons (TRPC6) whereas an increase in TRPC 3 expression may be required for dendritic tree development in Purkinje cells. The fact that TRPC proteins undergo marked changes in expression after birth, and in a neuron-specific manner, suggests that this novel family of ion channels may be intimately involved in cerebellar development.

We show that activation of group I mGluRs, which couple to PLC activation, gives rise to increases in intracellular $\mathrm{Ca}^{2+}$ concentration in Purkinje cell soma and dendrites under conditions when intracellular $\mathrm{Ca}^{2+}$ stores are depleted and voltage-gated channels cannot activate. This means that the observed increase in intracellular $\mathrm{Ca}^{2+}$ concentration following activation of group I mGluRs likely reflects $\mathrm{Ca}^{2+}$ influx through TRPC channels. In theory, it is possible that TRPC channel activation depolarises the surrounding membrane sufficiently to activate voltage-gated $\mathrm{Ca}^{2+}$ channels which could then give rise to an increase in intracellular $\mathrm{Ca}^{2+}$ concentration. However, it is unlikely that this is the case here since cells were clamped at $-60 \mathrm{mV}$ and only $\mathrm{Ca}^{2+}$ signals from proximal dendrites, whose membrane potential should be controlled [9], were used for analysis. 


\section{Acknowledgements}

The authors are grateful to Prof. W. Schilling for providing TRPC antibodies and discussion and Prof. A. Parekh for helpful comments on the manuscript. The authors would also like to thank C. Nelson and Dr. P. Camelliti for their extensive help with

confocal microscopy and Dr. T. Dawson for technical assistance. This work was supported by the Royal Society. 


\section{References}

1. Ramsey IS, Delling M, Clapham DE. (2006) An introduction to TRP channels. Annual Review of Physiology, 68, 619-647.

2. Zufall F. (2005) The TRPC2 ion channel and pheromone sensing in the accessory olfactory system. Naunyn-Schmiedeberg's Archives of Pharmacology, 371, 245.

3. Putney JJW. (2004) The enigmatic TRPCs: multifunctional cation channels. Trends in Cell Biology, 14, 282.

4. Bezzerides VJ, Ramsey IS, Kotecha S, Greka A, Clapham DE. (2004) Rapid vesicular translocation and insertion of TRP channels. Nat Cell Biol, 6, 709.

5. Greka A, Navarro B, Oancea E, Duggan A, Clapham DE. (2003) TRPC5 is a regulator of hippocampal neurite length and growth cone morphology. Nat Neurosci, 6, 837.

6. Li Y, Jia Y-C, Cui K, Li N, Zheng Z-Y, Wang Y-z, Yuan X-b. (2005) Essential role of TRPC channels in the guidance of nerve growth cones by brain-derived neurotrophic factor. Nature, 434, 894.

7. Pla AF, Maric D, Brazer S-C, Giacobini P, Liu X, Chang YH, Ambudkar IS, Barker JL. (2005) Canonical Transient Receptor Potential 1 Plays a Role in Basic Fibroblast Growth Factor (bFGF)/FGF Receptor-1-Induced Ca2+ Entry and Embryonic Rat Neural Stem Cell Proliferation. J. Neurosci., 25, 2687-2701.

8. Sotelo C. (2004) Cellular and genetic regulation of the development of the cerebellar system. Progress in Neurobiology, 72, 295.

9. Glitsch M, Parra P, Llano I. (2000) The retrograde inhibition of IPSCs in rat cerebellar Purkinje cells is highly sensitive to intracellular $\mathrm{Ca} 2+$. European Journal of Neuroscience, 12, 987-993.

10. Llano I, Marty A, Armstrong C, Konnerth A. (1991) Synaptic- and agonistinduced excitatory currents of Purkinje cells in rat cerebellar slices. Journal of Physiology, 434, 183-213.

11. Mullen RJ, Buck CR, Smith AM. (1992) NeuN, a neuronal specific nuclear protein in vertebrates. Development, 116, 201-211. 
12. Weyer A, Schilling K. (2003) Developmental and cell type-specific expression of the neuronal marker NeuN in the murine cerebellum. Journal of Neuroscience Research, 73, 400-409.

13. Glitsch M. (2006) Selective Inhibition of Spontaneous But Not Ca2+-Dependent Release Machinery by Presynaptic Group II mGluRs in Rat Cerebellar Slices. J Neurophysiol, 96, 86-96.

14. Schaefer M, Plant TD, Stresow N, Albrecht N, Schultz G. (2002) Functional Differences between TRPC4 Splice Variants. J. Biol. Chem., 277, 3752-3759.

15. Goel M, Sinkins WG, Schilling WP. (2002) Selective Association of TRPC Channel Subunits in Rat Brain Synaptosomes. J. Biol. Chem., 277, 48303-48310.

16. Mizuno N, Kitayama S, Saishin Y, Shimada S, Morita K, Mitsuhata C, Kurihara H, Dohi T. (1999) Molecular cloning and characterization of rat trp homologues from brain. Molecular Brain Research, 64, 41.

17. Kim SJ, Kim YS, Yuan JP, Petralia RS, Worley PF, Linden DJ. (2003) Activation of the TRPC1 cation channel by metabotropic glutamate receptor mGluR1. Nature, 426, 285.

18. Sossey-Alaoui K, Lyon JA, Jones L, Abidi FE, Hartung AJ, Hane B, Schwartz CE, Stevenson RE, Srivastava AK. (1999) Molecular Cloning and Characterization of TRPC5 (HTRP5), the Human Homologue of a Mouse Brain Receptor-Activated Capacitative Ca2+ Entry Channel. Genomics, 60, 330.

19. Montell C, Birnbaumer L, Flockerzi V. (2002) The TRP Channels, a Remarkably Functional Family. Cell, 108, 595. 


\section{Figure legends}

Fig. 1: TRPC4 mRNA levels are significantly down-regulated during postnatal cerebellar development.

A, RT-PCR products are shown using primers for TRPC1, TRPC3-7 and GAPDH (internal RT-PCR control) with mRNA isolated at postnatal days 1, 4, 8, 12, 16, 21, 28 and 42 from whole rat cerebella. All TRPCs (except for TRPC2) are prominently expressed in postnatal rat cerebellum. There is a steady and pronounced decrease in TRPC4 mRNA expression during cerebellar development. B, Relative TPRC mRNA expression at postnatal days 1 (P1, black bar), 16 (P16, light grey bar) and 42 (42, dark grey bar), normalised to average expression value obtained at P1 for a given TRPC subunit (for quantification see methods; average of 3-4 independently run RT-PCR experiments). Only for TRPC4, is there a significant decrease in mRNA expression (46\% $\pm 9.3 \%$ reduction; $P=0.0126 ; n=3$; unpaired $\mathrm{t}$-test)

\section{Fig. 2: Significant changes in TRPC3, TRPC4 and TRPC6 protein expression during postnatal cerebellar development.}

A, Western blots for TRPC1, TRPC3-7 and GAPDH at postnatal days 1, 16 and 42 from whole rat cerebella. There is an increase in TRPC3 protein expression and a decrease in TRPC4 and TRPC6 protein expression. B, Relative TRPC protein expression at postnatal days 1 (P1, black bar), 16 (P16, light grey bar) and 42 (P42, dark grey bar), normalised to average expression value obtained at P1 for a given TRPC subunit (for quantification see methods; average of 3-4 independent Western blots). There is a significant increase in TRPC3 expression (145.4\% $\pm 6.4 \%$ increase; $P<0.0001 ; n=4$; unpaired t-test) and a significant decrease in both TRPC4 and TRPC6 expression (TRPC4: $67.1 \% \pm 10.6 \%$ decrease; $P=0.0025 ; n=4$; unpaired t-test; TRPC6: $49.3 \% \pm 5.7 \%$ decrease; $P=0.0045$; $n=3$; unpaired t-test). 
Fig. 3: TRPC3 expression is mainly found on cerebellar Purkinje cell bodies and their dendrites.

Immunohistochemical analysis of rat cerebellar slices at postnatal days 1 (P1, left), 16 (P16, middle) and 42 (P42, right) with anti-TRPC3 antibodies (Professor Schilling). At $\mathrm{P} 1$, there is diffuse staining of the slice which is strongest in the Purkinje cell layer and weakest in the external/molecular layer. At P16, TRPC3 staining is prominent on Purkinje cell bodies in the Purkinje cell layer (PCL) and their dendrites in the molecular layer (ML) but absent in the external granule cell layer and only weak in the internal granule cell layer (IGL). At P42, TRPC3 staining is still prominent on Purkinje cell bodies and their dendrites in the ML and only weak in the IGL.

\section{Fig. 4: TRPC4 expression mirrors cerebellar granule cell development.}

Immunohistochemical analysis of rat cerebellar slices at postnatal days 1 (P1, left), 16 (P16, middle) and 42 (P42, right) with anti-TRPC4 antibodies (Chemicon). At P1, TRPC4 staining is found in the external granule cell layer (EGL). At P16, TRPC4 staining is found in the EGL, the molecular layer (ML) and the internal granule cell layer (IGL). At P42, TRPC4 staining is restricted to the IGL.

Fig. 5: NeuN, a specific marker for post-mitotic granule cells, and TRPC4 are confined to the same cell population.

Immunohistochemical analysis of rat cerebellar slices at postnatal day 28 (P28) with antiTRPC4 antibodies (Chemicon; left, green fluorescence) and anti-NeuN antibodies (right, red fluorescence). Both antibodies only stain cells in the internal granule cell layer (IGL). There is no staining of the molecular layer (ML) or the Purkinje cell layer (PCL).

Fig. 6: TRPC6 expression is found on Purkinje cell bodies, granule cells and interneurons.

Immunohistochemical analysis of rat cerebellar slices at postnatal days 1 (P1, left), 16 (P16, middle) and 42 (P42, right) with anti-TRPC6 antibodies (Prof. William Schilling). At P1, TRPC6 staining is diffuse with more staining in the Purkinje cell layer. At P16, TRPC6 staining is found on Purkinje cell bodies in the Purkinje cell layer (PCL), on 
granule cells in the internal granule cell layer (IGL) and on cells in the molecular layer (ML). At P42, TRPC6 staining is identical to that at P16: Staining of Purkinje cell bodies, cells in the ML and granule cells in the IGL. Since at this stage of development, there are only interneurons in the ML, the TRPC6 positive cells at both P16 and P42 are likely to be interneurons.

\section{Fig. 7: Activation of group I mGluRs leads to increases in intracellular $\mathrm{Ca}^{2+}$ concentration that are independent of intracellular $\mathrm{Ca}^{2+}$ stores and voltage-gated conductances and likely reflect $\mathrm{Ca}^{2+}$ entry through TRPC channels.}

Results from one representative Purkinje cell which was clamped at $-60 \mathrm{mV}$ and which was loaded with $400 \mu \mathrm{M}$ Fura-2 to measure intracellular $\mathrm{Ca}^{2+}$ concentration changes. Slice was incubated in $2 \mu \mathrm{M}$ thapsigargin and $10 \mu \mathrm{M}$ ryanodine for $60 \mathrm{~min}$ prior to experiment to deplete intracellular $\mathrm{Ca}^{2+}$ stores. Experiments were carried out in the continued presence of both thapsigargin and ryanodine and in the additional presence of $0.3 \mu \mathrm{M}$ TTX and $10 \mu \mathrm{M}$ CNQX. Application of $100 \mu \mathrm{M}$ DHPG resulted in an increase in intracellular $\mathrm{Ca}^{2+}$ concentration both in the soma (top panel; solid black line) and proximal dendrites (top panel; dotted black lines) while there was a concurrent inward current (bottom panel). Both intracellular $\mathrm{Ca}^{2+}$ increase and inward current likely reflect activation of TRPC channels because these channels are activated following stimulation of PLC (via group I mGluRs) and neither require $\mathrm{Ca}^{2+}$ release from intracellular $\mathrm{Ca}^{2+}$ stores nor a change in membrane potential for activation. Similar results were observed in two more cells.

Changes in intracellular $\mathrm{Ca}^{2}$ concentration are depicted as changes in $? \mathrm{~F}_{356} / \mathrm{F}_{380}$ and are given in arbitrary units (u). 
Figure 1

A

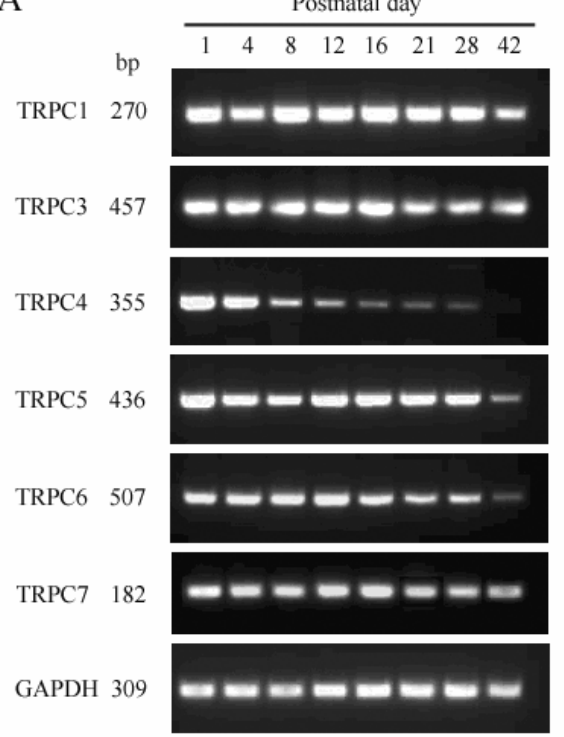

B

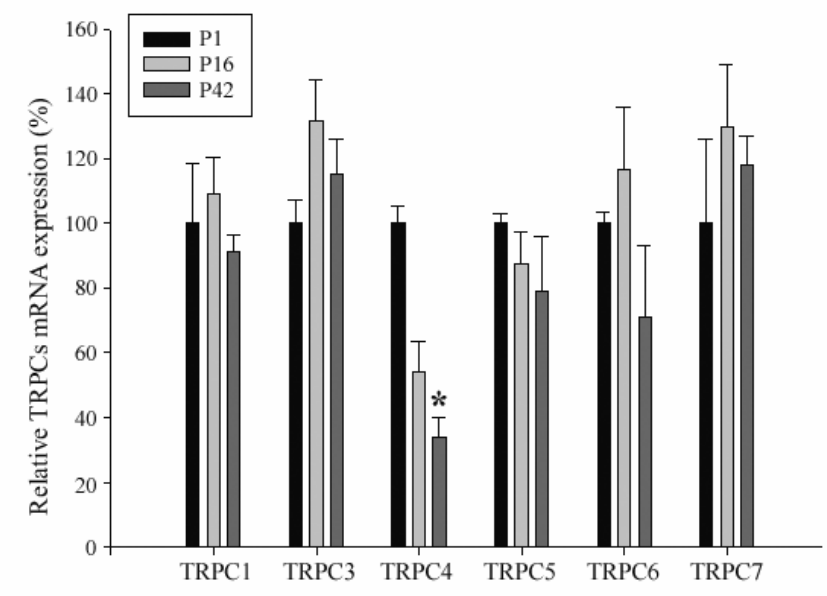

Figure 2

A

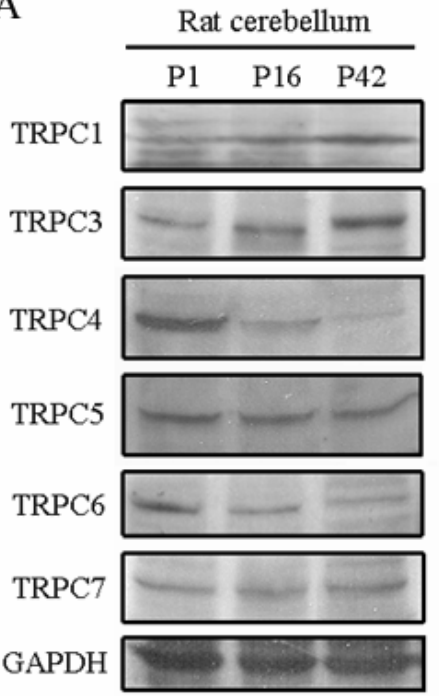

B

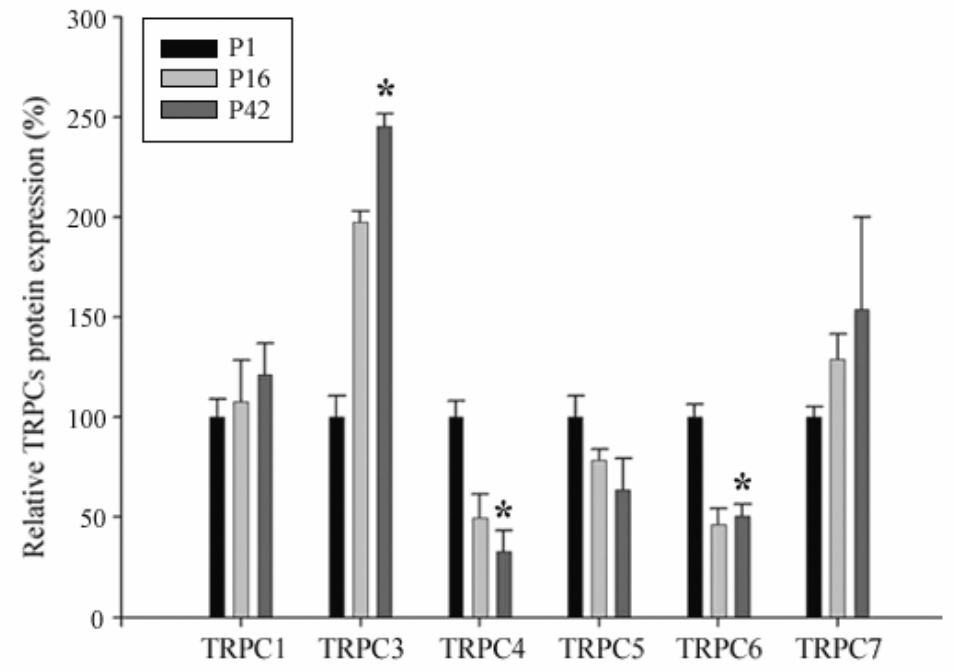


Figure 3

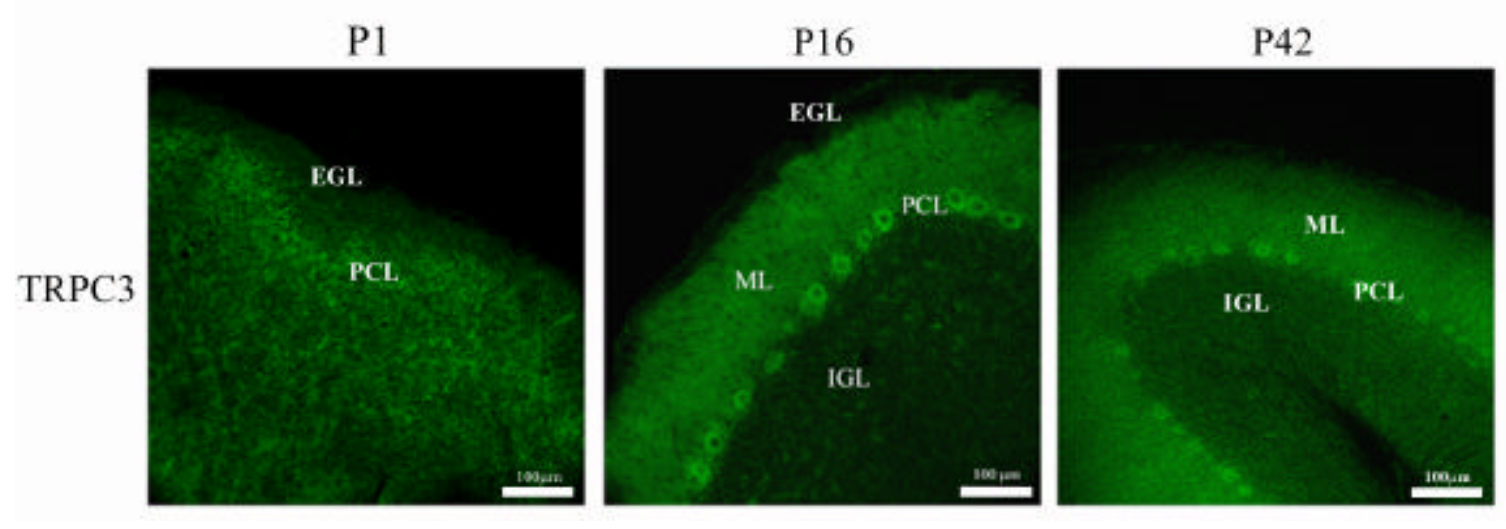

Figure 4

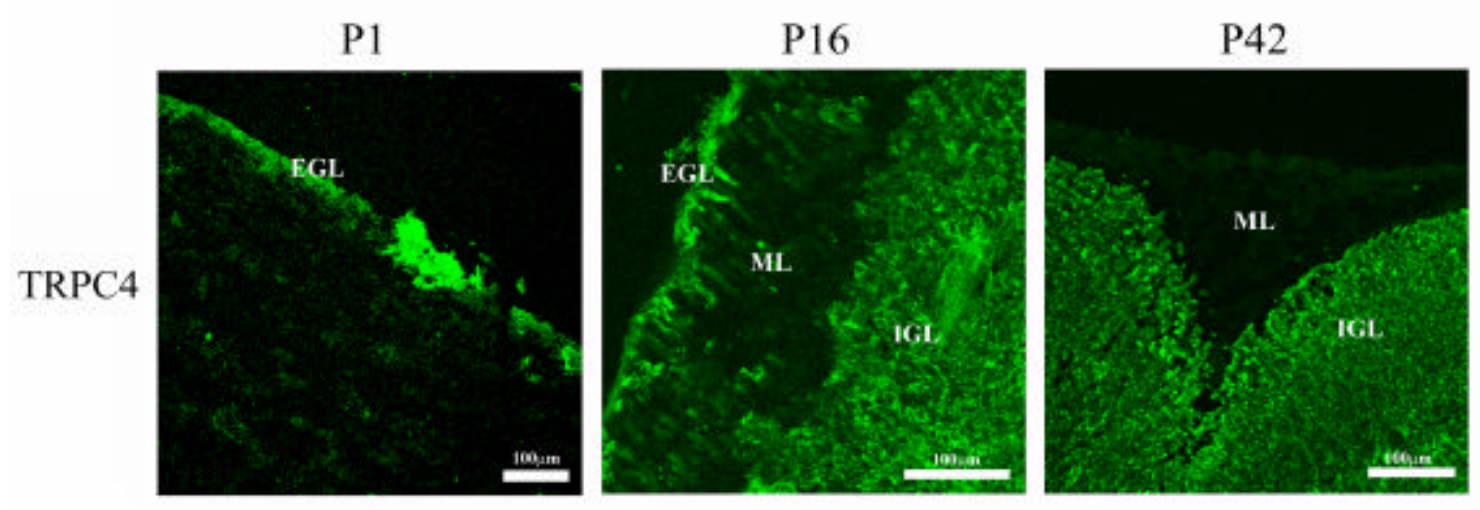




\section{Figure 5}
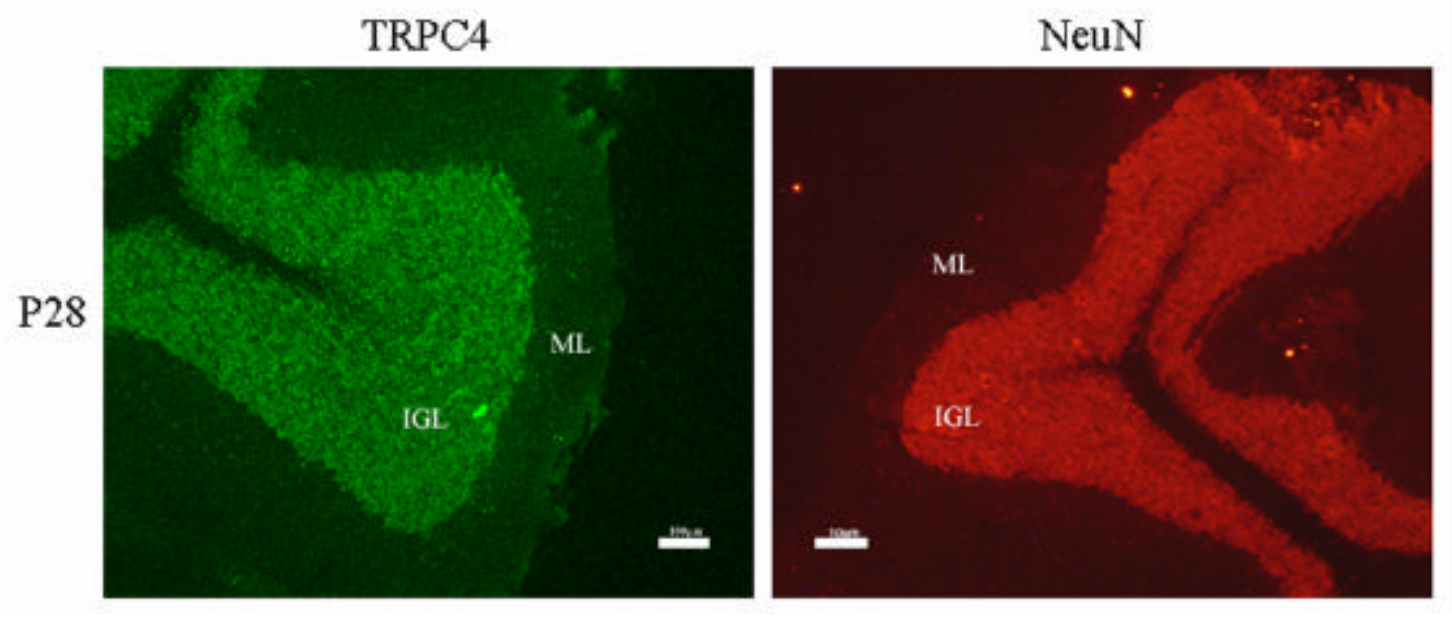

Figure 6

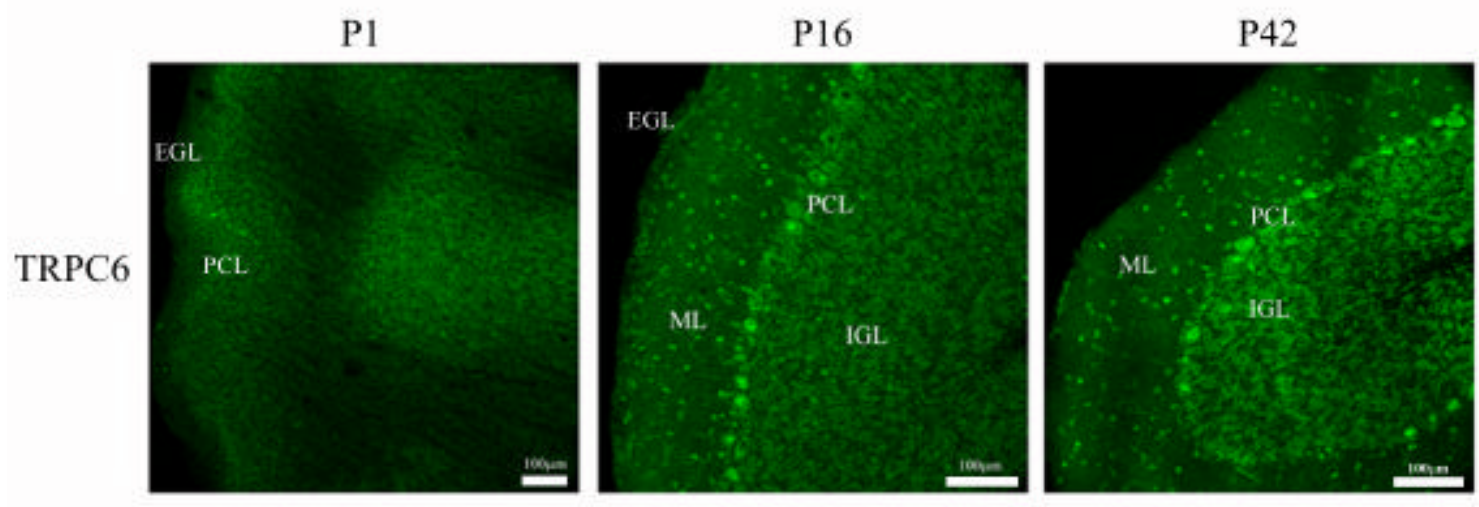


Figure 7
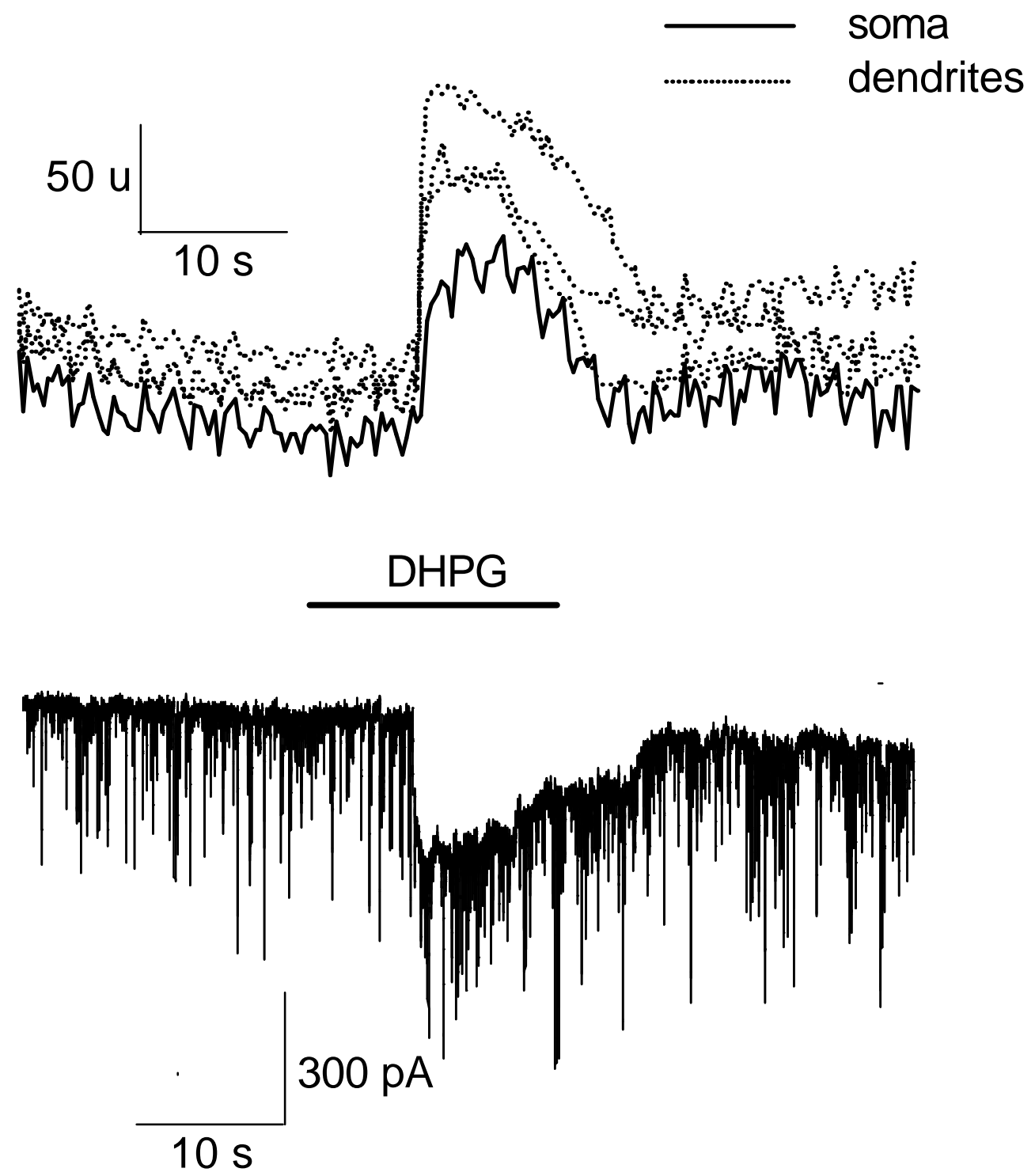